\title{
In situ detection of SARS-CoV-2 in lungs and airways of patients with COVID-19
}

\author{
Inga-Marie Schaefer $\mathbb{D}^{1} \cdot$ Robert F. Padera ${ }^{1} \cdot$ Isaac H. Solomon $\mathbb{1}^{1} \cdot$ Sanjat Kanjilal ${ }^{2,3} \cdot$ Mark M. Hammer ${ }^{4} \cdot$ \\ Jason L. Hornick ${ }^{1} \cdot$ Lynette M. Sholl $\mathbb{B}^{1}$
}

Received: 22 May 2020 / Revised: 5 June 2020 / Accepted: 5 June 2020 / Published online: 19 June 2020

(c) The Author(s), under exclusive licence to United States \& Canadian Academy of Pathology 2020

\begin{abstract}
Coronavirus disease 2019 (COVID-19) caused by SARS-CoV-2 has led to a global public health crisis. In elderly individuals and those with comorbidities, COVID-19 is associated with high mortality, frequently caused by acute respiratory distress syndrome. We examine in situ expression of SARS-CoV-2 in airways and lung obtained at autopsy of individuals with confirmed COVID-19 infection. Seven autopsy cases (male, $N=5$; female, $N=2$ ) with reverse transcriptase-polymerase chain reaction (RT-PCR)-confirmed SARS-CoV-2 infection and a median age of 66 years (range, 50-77 years) were evaluated using a rabbit polyclonal antibody against SARS Nucleocapsid protein in correlation with clinical parameters. The median time from symptom onset to death was 9 days (range, 6-31 days), from hospitalization 7 days (range, 1-21 days), from positive RT-PCR 7 days (range, 0-18 days), and from intensive care unit admission defining onset of respiratory failure 3 days (range, $1-18$ days). Chest imaging identified diffuse airspace disease in all patients corresponding to acute and $(N=5)$ or organizing $(N=2)$ diffuse alveolar damage (DAD) on histologic examination. Among five patients with acute-phase DAD ( $\leq 7$ days from onset of respiratory failure), SARS-CoV-2 was detected in pulmonary pneumocytes and ciliated airway cells $(N=5)$, and in upper airway epithelium $(N=2)$. In two patients with organizing DAD ( $>14$ days from onset of respiratory failure), no virus was detected in lungs or airways. No endothelial cell infection was observed. The findings suggest that SARS-CoV-2 infection of epithelial cells in lungs and airways of patients with COVID-19 who developed respiratory failure can be detected during the acute phase of lung injury and is absent in the organizing phase.
\end{abstract}

\section{Introduction}

Severe acute respiratory syndrome coronavirus 2 (SARSCoV-2) (https://www.who.int/csr/don/05-january-2020-

Supplementary information The online version of this article (https:// doi.org/10.1038/s41379-020-0595-z) contains supplementary material, which is available to authorized users.

Lynette M. Sholl

lmsholl@bwh.harvard.edu

1 Department of Pathology, Brigham and Women's Hospital, Harvard Medical School, Boston, MA, USA

2 Division of Infectious Diseases, Brigham and Women's Hospital, Boston, MA, USA

3 Department of Population Medicine, Harvard Medical School and Harvard Pilgrim Healthcare Institute, Boston, MA, USA

4 Department of Radiology, Brigham and Women's Hospital, Harvard Medical School, Boston, MA, USA pneumonia-of-unkown-cause-china/en/) [1], the virus responsible for the Coronavirus disease 2019 (COVID-19), emerged in Wuhan, China in early December 2019 (https://www.who.int/csr/don/05-january-2020-pneumoniaof-unkown-cause-china/en/) and achieved pandemic status 3 months later. COVID-19 is associated with a case fatality rate of $1-10 \%$, with greater mortality in elderly patients and those with cardiovascular and pulmonary comorbidities [2-4]. Most patients present with fever and cough; a subset progress to severe acute respiratory distress syndrome (ARDS) [2-4].

Lung histopathologic manifestations of COVID-19 described to date are largely nonspecific and include edema, proteinaceous exudate, diffuse alveolar damage (DAD) with hyaline membrane formation, reactive pneumocyte hyperplasia, interstitial mononuclear inflammatory infiltrates dominated by lymphocytes, and multinucleated giant cells, often without telltale viral cytopathic changes or characteristic intranuclear or intracytoplasmic viral inclusions [5-11]. Vascular thrombosis, however, appears to be 
more common in COVID-19 lungs [12]. However systematic descriptions of the gross and histopathologic findings in the lower respiratory tract are still limited.

Molecular and serologic testing methods for COVID-19 diagnosis and seroconversion are the focus of many ongoing studies [13-17]. Based on the distribution of viral RNA in clinical specimens, SARS-CoV-2 is thought to infect the nasopharynx and then spread to the lower respiratory tract $[18,19]$; however, the temporal dynamics of viral tropism in patients infected with SARS-CoV-2 have not been illustrated in situ in patient samples.

Anti-SARS immunohistochemical (IHC) testing on formalin-fixed paraffin-embedded (FFPE) tissue sections obtained through surgical resection or autopsy can aid in identifying histologic correlates of SARS-CoV-2 infection and in establishing a specific tissue diagnosis.

The aim of this study was to examine the gross and histologic patterns of tissue injury in correlation with viral protein expression in the conducting airways and lungs at autopsy in a series of patients with confirmed SARS-CoV-2 infection.

\section{Materials and methods}

\section{Patient samples}

This study was performed with the approval of the Institutional Review Board at Brigham and Women's Hospital. Cases were retrieved from the Anatomic Pathology files of Brigham and Women's Hospital and included seven patients with laboratory confirmed COVID-19 who underwent autopsy in April 2020. All patients had tested positive for SARS-CoV-2 by RT-PCR of nasopharyngeal swabs in a CLIA-certified laboratory during hospital admission. All patients had radiologic imaging performed and underwent unrestricted autopsy with complete anatomic dissection, except for patient 5 in whom the brain was excluded from the autopsy permission. Hematoxylin and eosin-stained histologic sections were reviewed by multiple surgical pathologists (IMS, RFP, IHS, JLH, and LMS).

\section{Immunohistochemistry (IHC)}

IHC was performed on 4- $\mu$ m-thick FFPE tissue sections following pressure cooker antigen retrieval (Target Retrieval Solution; pH 6.1; Dako, Carpinteria, CA) using a rabbit polyclonal antibody directed against a peptide derived from SARS-CoV Nucleocapsid protein [20] (catalog no. NB10056576, Novus Biologicals, Centennial, CO; 1:500 dilution) and the Novolink Polymer Detection System (Leica Biosystems, Buffalo Grove, IL) creating a brown reaction product. For double labeling of SARS with TTF-1 and PU.1
(SPI1), respectively, a red reaction product was used for SARS (1:200 dilution), together with mouse monoclonal antibodies directed against TTF-1 (clone 8G7G3/1, catalog no. M3575, Dako, Carpinteria, CA; 1:200 dilution) and PU.1 (clone G148-74, catalog no. 554268, BD Biosciences, San Jose, CA; 1:200 dilution), with the EnVision Plus detection system (Dako) resulting in a brown reaction product. For double labeling of SARS with p63, MUC5AC, and FOXJ1, a red reaction product was used for SARS (1:200 dilution), together with mouse monoclonal antibodies against p63 (clone 4A4, Biocare Medical, Pacheco, CA; 1:150 dilution), MUC5AC (clone 45M1, Abcam, Cambridge, MA; 1:10,000 dilution), and FOXJ1 (clone CL3989, Sigma, St. Louis, MO; 1:250 dilution), with the EnVision Plus detection system (Dako) resulting in a brown reaction product. Appropriate positive (i.e., lung samples from individuals with COVID-19 obtained at autopsy) and negative controls (i.e., lung samples from individuals preCOVID-19 pandemic) were used throughout. The SARS$\mathrm{CoV}$ nucleocapsid antibody used in the present study has been evaluated by The Centers for Disease Control and Prevention (CDC) and the authors demonstrated no crossreactivity with influenza $A(\mathrm{H} 1 \mathrm{~N} 1)$, influenza $\mathrm{B}$, respiratory syncytial virus, parainfluenza virus type 3 , human coronavirus (HCoV) 229E, or MERS-CoV in PCR-confirmed tissues [21]. It has also been used in non-human primate studies [22] and overall appears to be highly sensitive and specific for SARS-CoV-2 during the ongoing pandemic. Three representative lung sections and one upper airway section were stained in each patient. The number of positive cells per $4 \mathrm{~mm}^{2}$ was counted to determine extent of staining. IHC stains were reviewed by multiple surgical pathologists (IMS, RFP, JLH, and LMS).

\section{Results}

\section{Clinicopathologic findings}

Clinicopathologic data in seven patients with COVID-19 (SARS-CoV-2 infection) who underwent autopsy are summarized in Table 1. Altogether, five male and two female patients with a median age of 66 years (range, 50-77 years) were evaluated. Presenting symptoms included cough in six patients, fever in five patients, dyspnea in three patients, hypoxemic respiratory failure in two patients, as well as cardiac arrest, diarrhea, hematuria, and altered mental status and increased lethargy in one case each (Table 1). Smoking status was known for $6 / 7$ patients and included 3 nonsmokers, 2 former smokers, and a current smoker.

The median time from symptom onset to death was 9 days (range, 6-31 days), from hospitalization to death 7 days (1-21 days), from intensive care unit (ICU) 


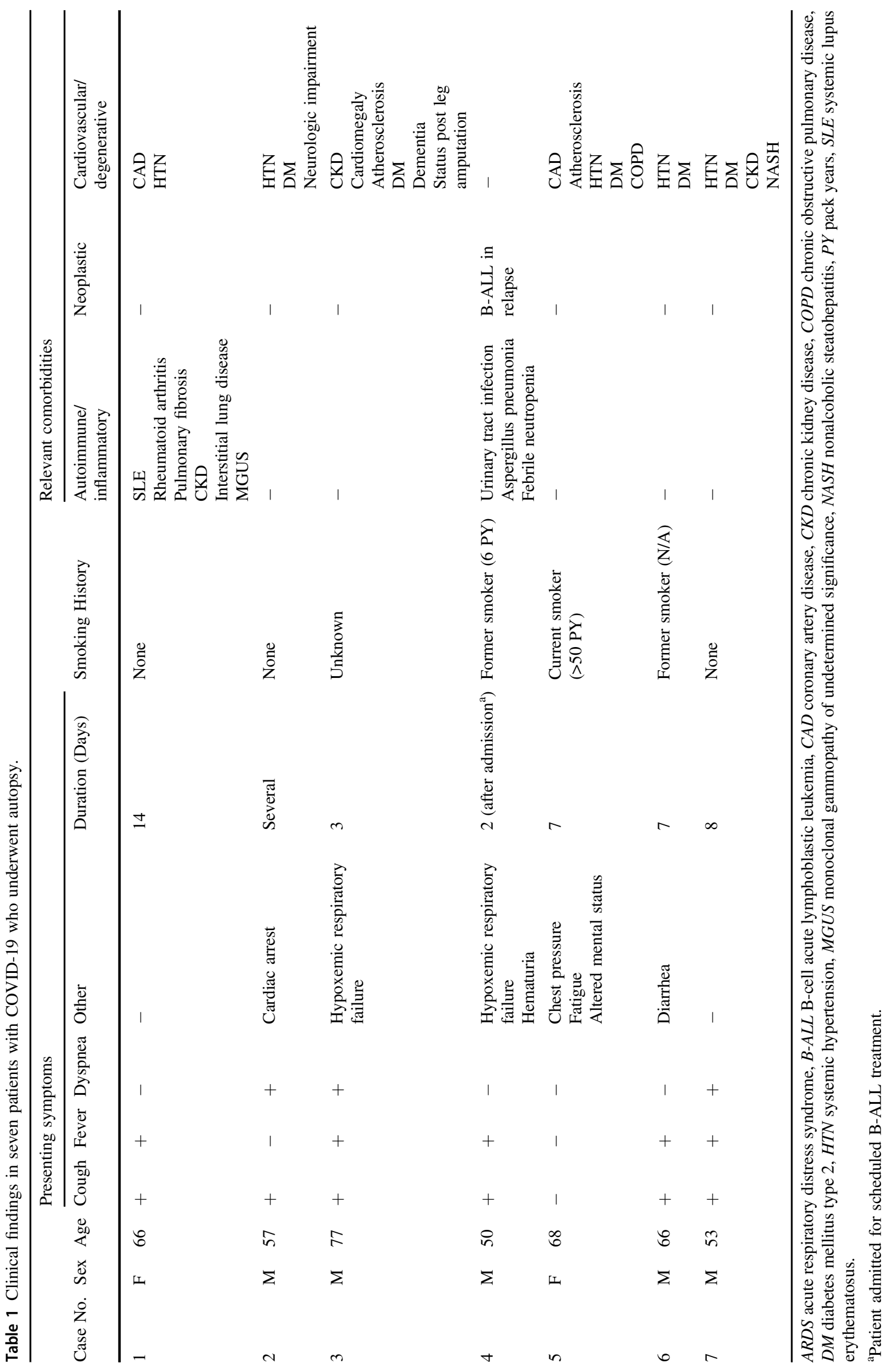




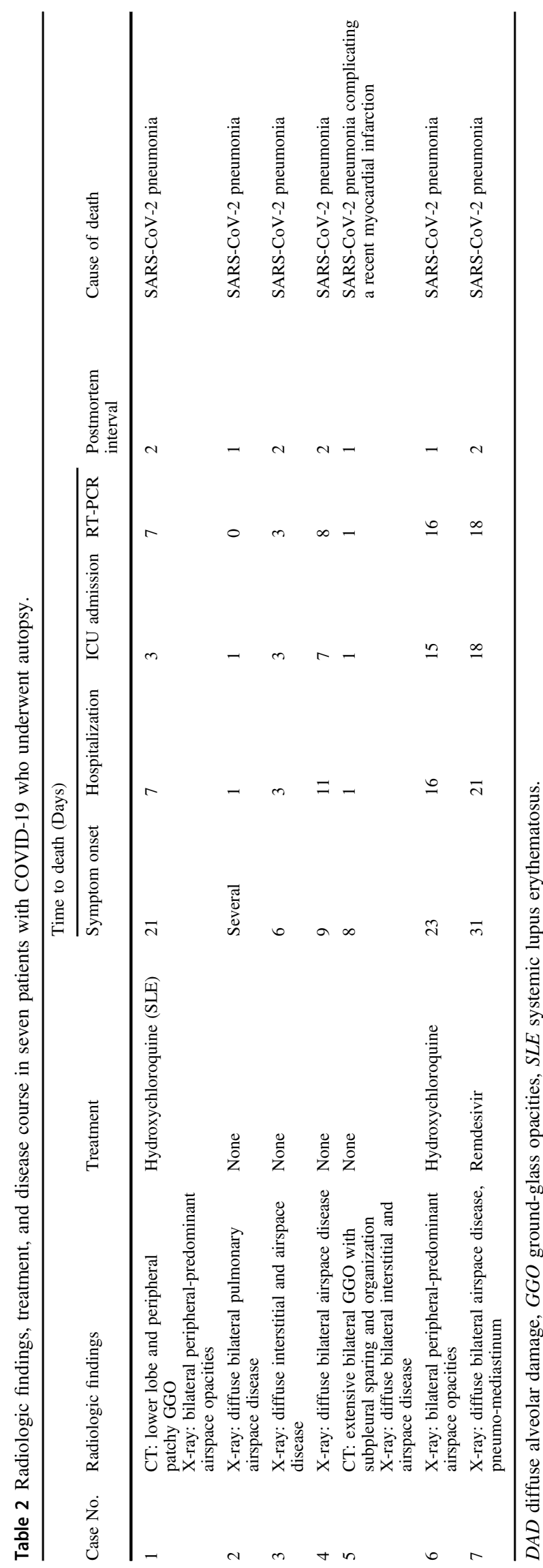

admission 3 days (1-18 days), and from positive SARSCoV-2 RT-PCR result to death 7 days (0-18 days) (Table 2 and Fig. 1). Of note, patients 2 and 3 had cognitive impairment and the duration of preadmission symptoms was unclear. Patient 4 was admitted without symptoms for scheduled treatment of B-lymphoblastic leukemia and developed cough and fever 2 days after admission. Patient 7 was admitted, discharged, and readmitted 3 days later. Relevant comorbidities were present in each of the seven patients and included autoimmune and inflammatory conditions in two patients, neoplastic conditions in one case, and cardiopulmonary and/or vascular diseases in six patients (Table 1).

All seven patients had abnormal findings on radiologic imaging (Table 2, Figs. 2 and 3), specifically diffuse bilateral airspace disease on chest $\mathrm{x}$-ray in each case, and ground-glass opacities on computed tomography (CT) in cases 1 and 5 (Fig. 2). Two patients were treated with hydroxychloroquine (in patient 1, however, prescribed for systemic lupus) and one patient received remdesivir (Table 2).

\section{Gross and histopathologic findings at autopsy}

The postmortem interval was 1-2 days in all cases (Table 2). The cause of death for all seven patients was SARS-CoV-2 pneumonia resulting in respiratory failure (Table 2 and Fig. 2); patient 5 had concurrent cardiac failure from a recent myocardial infarction.

Histologic examination of the lungs demonstrated acute DAD (Table 3) with prominent hyaline membranes in patients 1-5. Specifically, acute-phase DAD with scattered foci of organization was observed in cases 1-4 (Fig. 4). Acute-phase DAD alone was identified in case 5 (Fig. 2). Diffuse inflammatory infiltrates consisting of interstitial and peribronchial lymphocytes and intraalveolar macrophages were identified in each of the seven patients (Fig. 4). Hyperplastic alveolar lining cells were most pronounced in patients 4 and 5 (Table 3 and Supplementary Fig. 1); these cases showed diffuse TTF-1 positive desquamated pneumocytes and the characteristic cytology of DAD, including nucleomegaly, chromatin clumping, prominent nucleoli, and cytoplasmic vacuolation with basophilic stippling and occasional cytoplasmic hyaline. No definitive viral cytopathic changes were observed. Patients 6 and 7 showed organizing DAD only with obliteration of alveolar spaces and extensive scarring, and without evidence of acute-phase DAD (Fig. 5).

Evidence of coagulopathy (i.e., pulmonary embolism or in situ thrombi) was identified in patients $1,2,3,5$, and 7 (Table 3 and Fig. 6). Patient 1, who had a clinical history of SLE and rheumatoid arthritis, had changes consistent with known underlying connective tissue disease-associated 
Time course

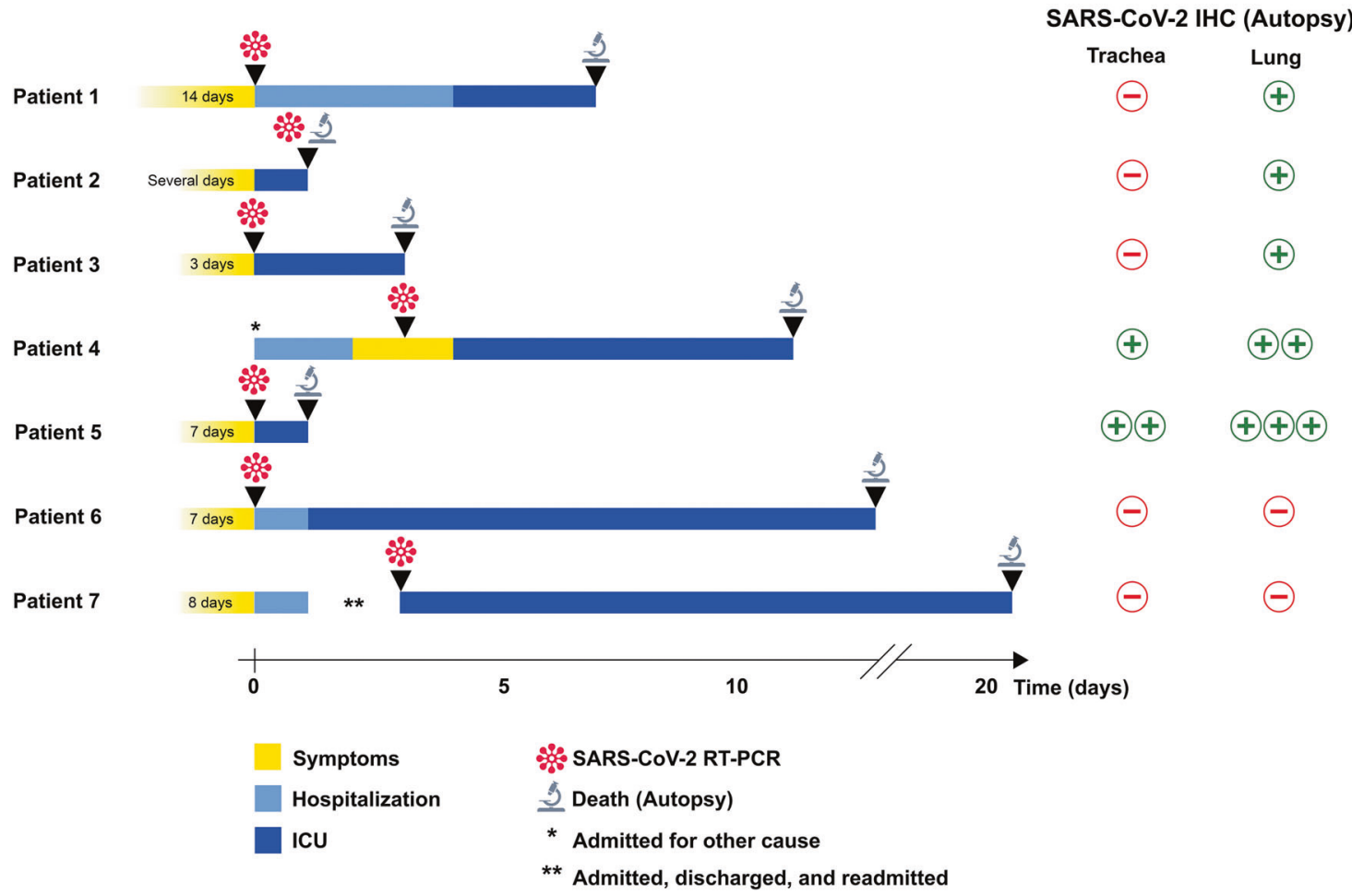

Fig. 1 Clinical time course in seven patients with COVID-19 (SARS-CoV-2 infection). The schematic illustrates symptom duration, hospitalization, admission to intensive care unit (ICU), detection of SARS-CoV-2 by reverse transcriptase-polymerase chain reaction (RT-PCR), and death followed by autopsy examination. *Patient 4 was admitted for scheduled B-lymphoblastic leukemia treatment and

interstitial lung disease. Patients 3, 4, and 7 exhibited superimposed bacterial lobar pneumonia, and patient 4 a preexisting chronic Aspergillus lung abscess. Tracheobronchial sections demonstrated reactive squamous metaplasia and postmortem epithelial detachment typical of autopsy tissue in all patients. Minimal lymphocytic infiltrates were present within edematous connective tissue of the airway wall.

\section{Immunohistochemical (IHC) findings at autopsy}

SARS IHC on lung sections detected SARS nucleocapsid protein within intraalveolar cells in patients 1-5 (Table 3, Figs. 4 and 7), and no staining in any of the lung sections from patients 6 to 7 (Table 3 and Fig. 5). In addition, in patients 1-5 weak positivity was detected within edema, hyaline membranes, and cell debris in alveolar spaces. Background autolysis was minimal and did not appear to affect IHC sensitivity. While the intensity of staining was equally strong in each of the five cases, the distribution of positive cells varied substantially: in patients $1-3,<5$ cells with positive staining were identified per $4 \mathrm{~mm}^{2}$ with comparable intensity across the three sections (Fig. 4). In developed symptoms 2 days after admission. **Patient 7 was hospitalized with symptoms, discharged, and readmitted 3 days later. Results of SARS immunohistochemistry (IHC) on autopsy trachea and lung tissue are shown on the right; "+" indicates positive staining in $<5$ cells per $4 \mathrm{~mm}^{2}$, " ++ " indicates positive staining in $5-50$ cells per $4 \mathrm{~mm}^{2}$, and " +++ " positive staining in $>50$ cells per $4 \mathrm{~mm}^{2}$.

contrast, patient 4 showed positive staining in 5-50 cells per $4 \mathrm{~mm}^{2}$ (Fig. 4), and patient 5 demonstrated positivity in $>50$ cells per $4 \mathrm{~mm}^{2}$ (Fig. 7). In patients 4 and 5 , positive staining was observed diffusely throughout the three sections including areas of reactive pneumocyte hyperplasia (Supplementary Fig. 1). Double labeling for SARS/TTF-1 and SARS/PU.1 was performed in lung sections of cases 4 and 5 (Fig. 7) and demonstrated that positive staining for SARS largely co-localized with TTF-1 positivity in pneumocytes. In contrast, the SARS/PU.1 double stain colocalized the markers in only rare intraalveolar macrophages of patient 5 (Fig. 7); of note, the intensity of staining in these cells was much less than that in the pneumocytes. There was no definitive SARS IHC reactivity in pulmonary endothelial cells.

SARS IHC on tracheal sections detected SARS nucleocapsid protein in a subset of ciliated airway epithelial cells and focally in the serous cells of submucous glands in patients 4 and 5 (Fig. 7). No virus was detected in tracheal sections of the remaining patients. Double labeling with SARS and specific epithelial cell markers demonstrated that virus was exclusive to the ciliated cells in the surface epithelium; p63-positive basal cells and MUC5AC-expressing 
secretory cells showed no evidence of infection (Fig. 7). FOXJ1 highlighted uninfected ciliated cells but was absent
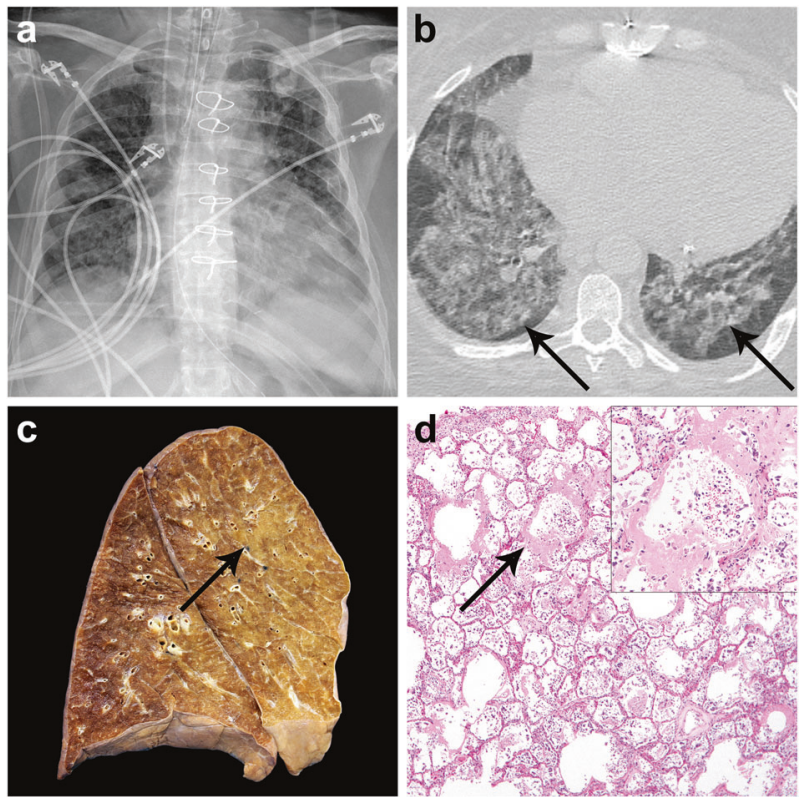

Fig. 2 Results of radiologic imaging and autopsy findings in patient 5 with COVID-19 (SARS-CoV-2 infection). Chest $x$-ray shows extensive interstitial and airspace opacities and cardiomegaly (a). CT imaging obtained the same day (b) demonstrates bilateral diffuse ground-glass opacities with subpleural sparing and linear bandlike opacities (b, arrows). Cross section of the left lung at autopsy (c) reveals patchy consolidation involving the upper and lower lobes representing SARS-CoV-2 pneumonia (c, arrow). Histologic examination of the lungs identified acute-phase diffuse alveolar damage (d) with extensive hyaline membrane formation (d, arrow) desquamation of pulmonary epithelial cells into the alveolar spaces, and inflammatory infiltrates $(\mathbf{d}$, inset). in SARS-expressing cells (Fig. 7). SARS nucleocapsid was also detected in bronchial and bronchiolar airways within the lung sections in patient 5 , to an extent similar to that observed in the upper airways (Supplementary Fig. 2). In case 4, no staining was identified in bronchial and bronchiolar airways within the lung, but evaluation was limited by extensive epithelial sloughing.

\section{Discussion}

In situ assays, such as tissue IHC, permit for precise cellular localization of infections in clinical samples. Our study indicates that SARS-CoV-2 infection is principally localized to the pneumocytes of the lung and ciliated cells of the airway [6, 21, 23], with negligible involvement of macrophages and no evidence of direct endothelial infection, in contrast to other reports [12, 20, 21, 24]. SARS-CoV-2 binds to the transmembrane angiotensin-converting enzyme 2 (ACE2) to enter type 2 pneumocytes, macrophages, pericytes, and cardiomyocytes [25]. Virally infected pneumocytes have been shown to produce interferon gamma and certain chemokine ligands leading to a macrophage hyperactivation program, uncontrolled inflammatory response, and severe lung injury [26]. Although prior studies have demonstrated SARS-CoV-2 nucleoprotein in tissue-resident macrophages, e.g., in lymph nodes and kidney, it is unclear if this reflects productive infection or phagocytosis of virally infected cellular debris [26]. In support of the latter, we observed marked difference in the levels of viral protein in infected lung epithelium and macrophages, suggesting distinct mechanisms of host cell-virus interactions.
Fig. 3 Radiologic imaging in six patients with COVID-19 (SARS-CoV-2 infection). Chest $\mathrm{X}$-rays in patients 1 (a), 2 (b), 3 (c), 4 (d), 6 (e), and 7 (f) showed extensive interstitial and airspace opacities consistent with COVID-19.
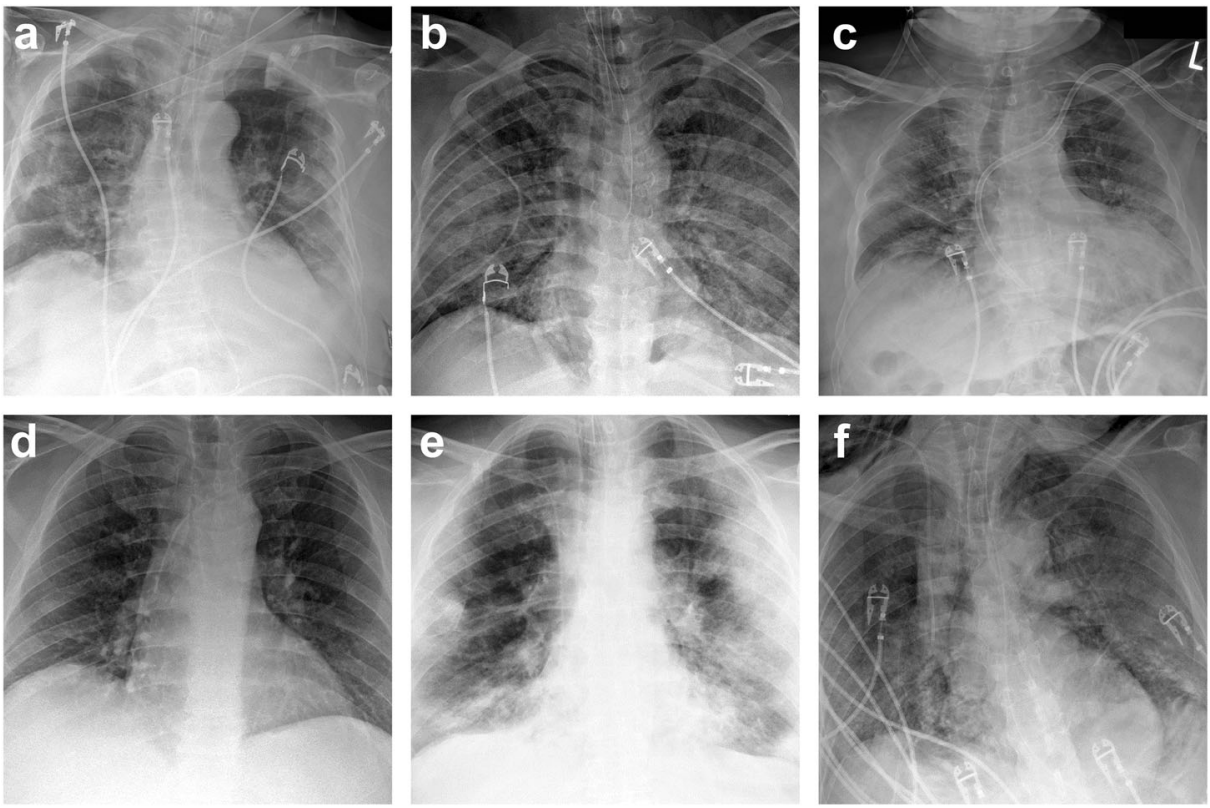


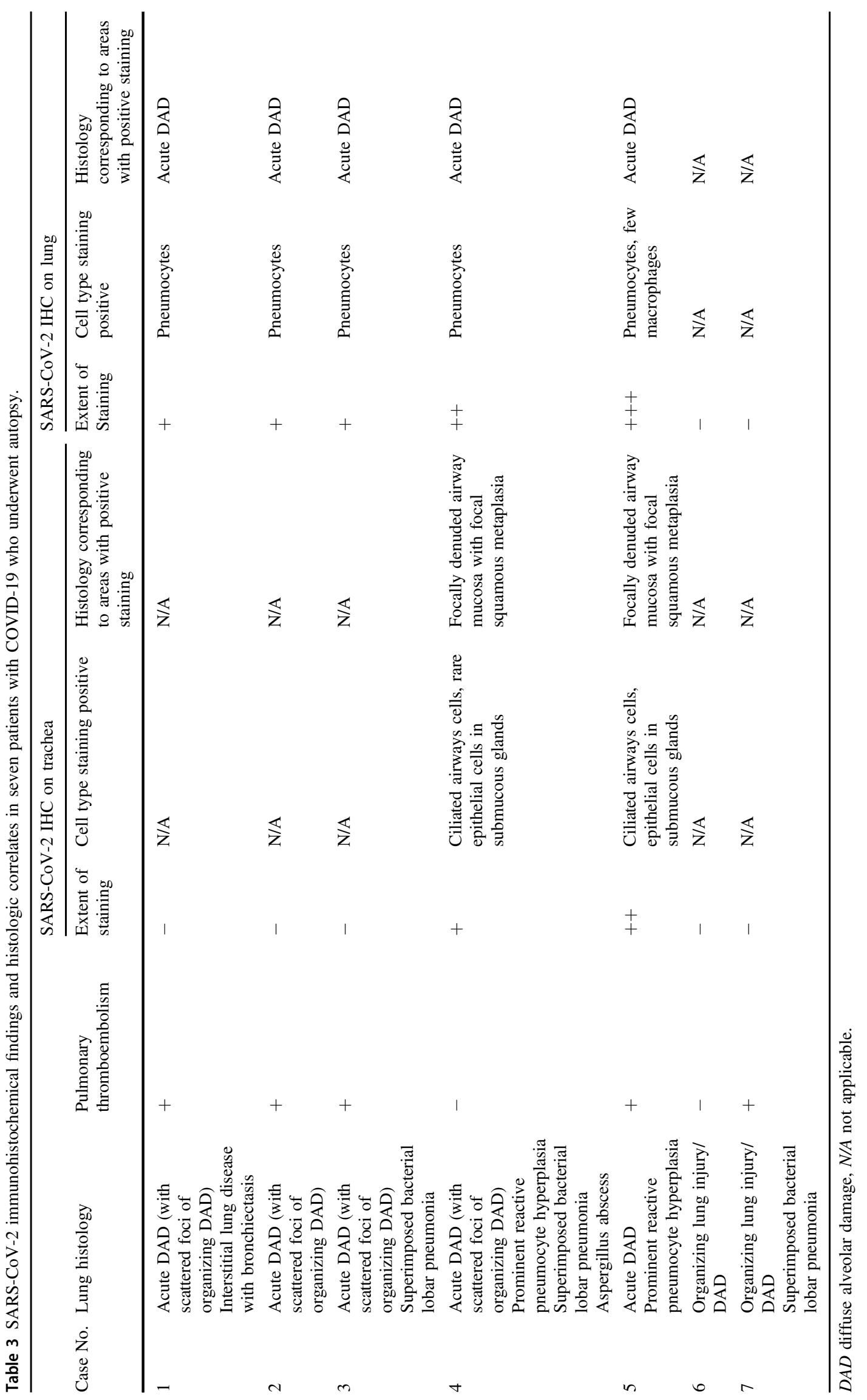




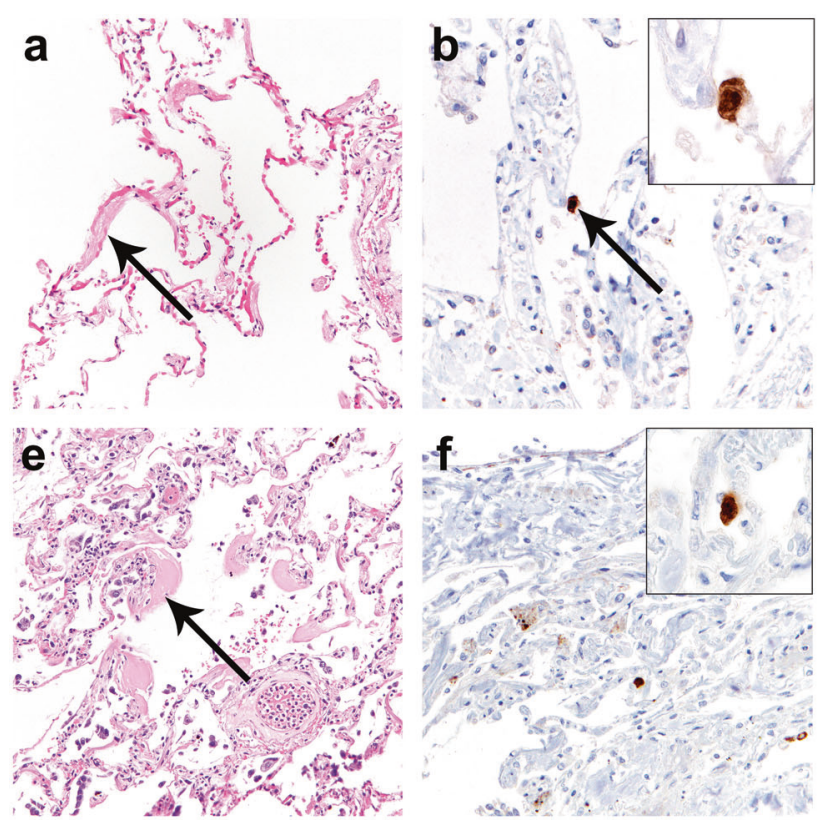

Fig. 4 Histologic lung findings at autopsy and SARS immunohistochemical staining in lungs from patients 1-4 with COVID-19 (SARS-CoV-2 infection). Histologic examination of the lungs from patients $1(\mathbf{a}, \mathbf{b}), 2$ (c, d), $3(\mathbf{e}, \mathbf{f})$, and $4(\mathbf{g}, \mathbf{h})$ identified acute-phase diffuse alveolar damage (DAD) with extensive hyaline membrane
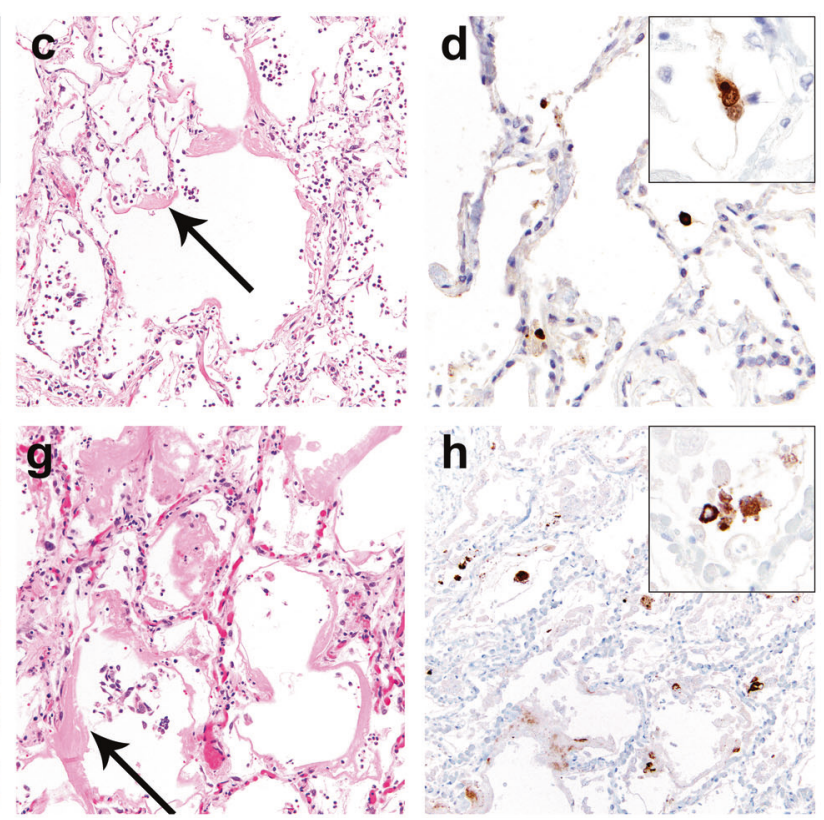

formation (a, c, e, f, arrows). SARS immunohistochemistry (IHC) showed $<5$ cells with strongly positive staining per $4 \mathrm{~mm}^{2}$ in patients 1 (b, arrow, inset), 2 (d, inset), and 3 (f, inset). In patient 4, 5-50 cells with strongly positive staining were identified per $4 \mathrm{~mm}^{2}$ (h, inset).

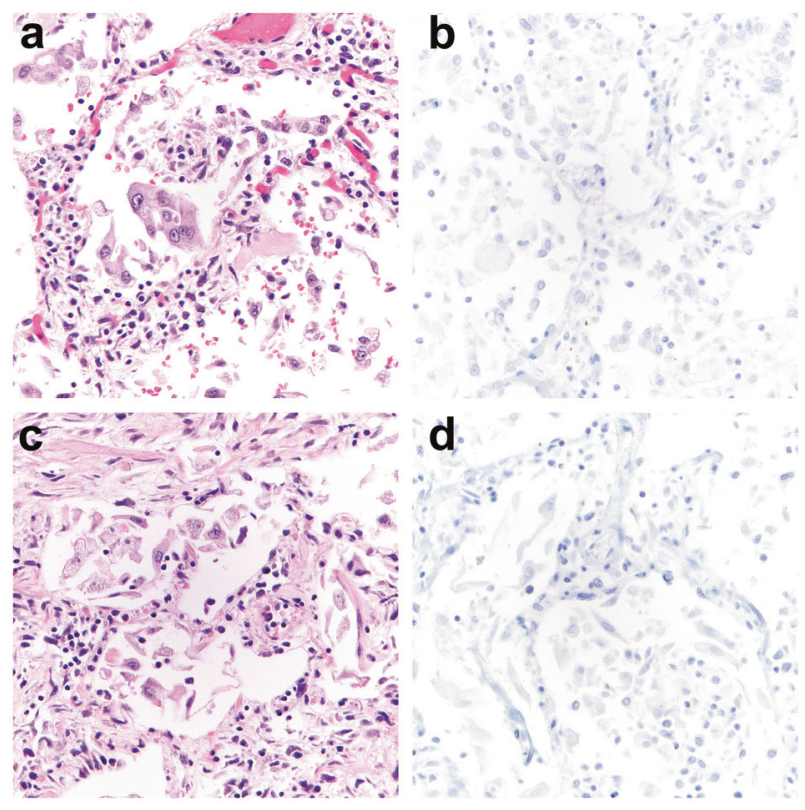

Fig. 5 Histologic lung findings at autopsy and SARS immunohistochemical staining in lungs from patients 6 and 7 with COVID-19 (SARS-CoV-2 infection). Histologic examination of the lungs from patients $6(\mathbf{a}, \mathbf{b})$, and 7 (c, d) showed organizing diffuse alveolar damage (DAD) (a, c) without prominent hyaline membrane formation that would correspond to acute-phase DAD. SARS immunohistochemistry (IHC) was entirely negative in lung sections from patient 6 (b) and 7 (d).

In our study, all cases with positive SARS IHC (cases 1-5) showed pneumocyte hyperplasia in lung sections.

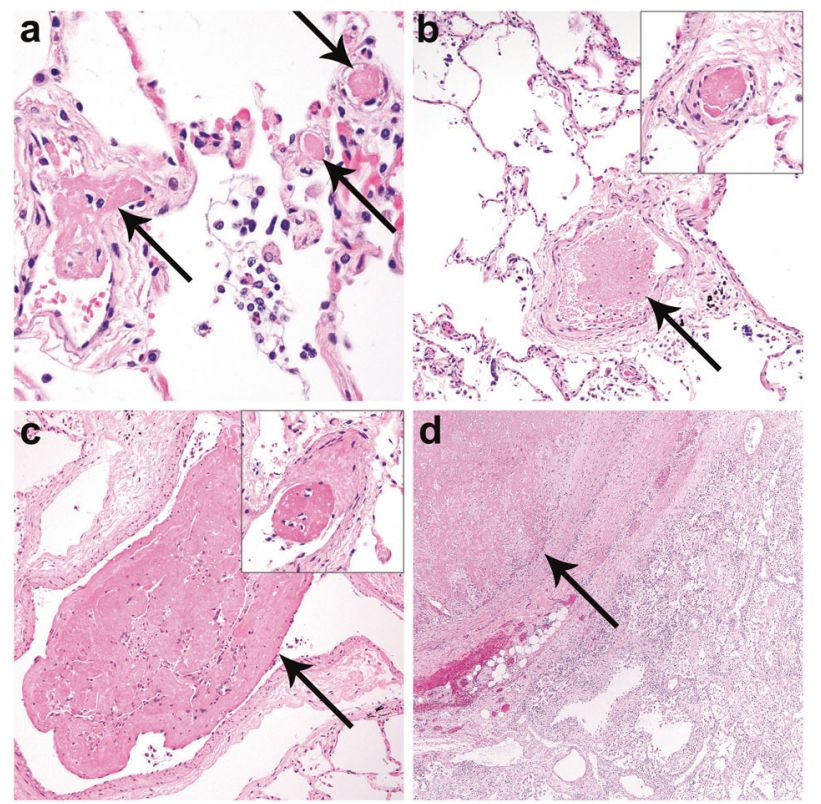

Fig. 6 Histologic evidence of coagulopathy in patients with COVID-19 (SARS-CoV-2 infection). Histologic examination of the lungs identified multiple thromboemboli in small to intermediate sized intrapulmonary arteries and post-capillary venules of patients 2 (a, arrows), 3 (b, arrow, inset), and 5 (c, arrow, inset) and a large thromboembolus in patient 7 (d, arrow).

This phenomenon is usually most pronounced at the end of the acute stage of DAD at $\sim$ day 7 but may be seen as early as 3 days and persists into the organizing 


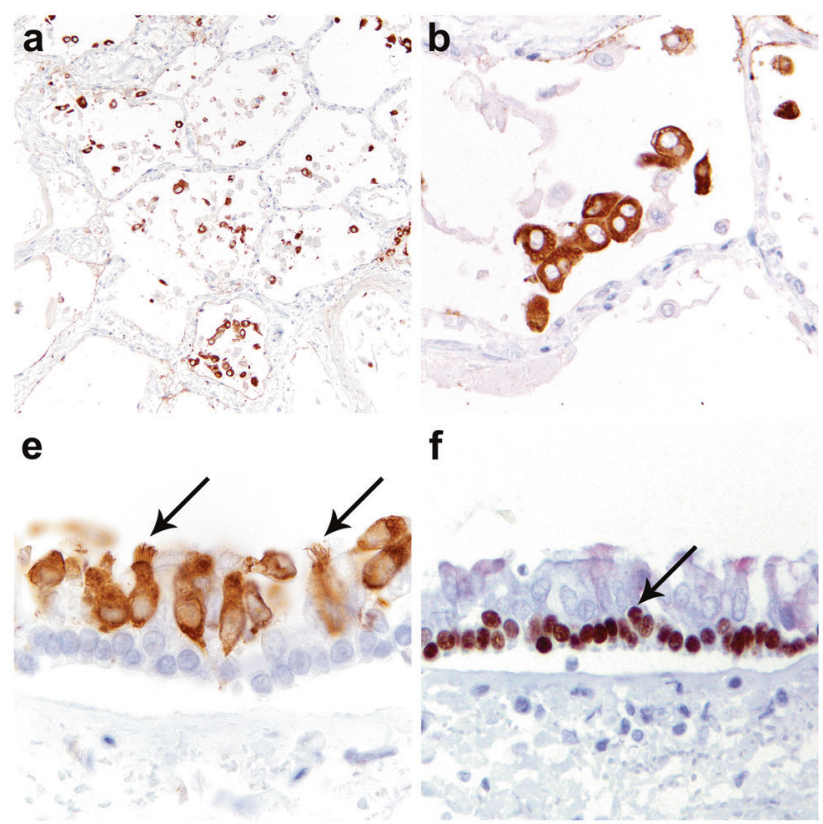

Fig. 7 SARS immunohistochemical staining in lung and airways from patient 5 with COVID-19 (SARS-CoV-2 infection). SARS immunohistochemistry (IHC) on lung sections shows diffuse strong SARS positivity in pneumocytes in $>50$ cells per $4 \mathrm{~mm}^{2}(\mathbf{a}, \mathbf{b})$. TTF-1/ SARS double labeling (c) demonstrates co-localization of TTF-1 (brown reaction product) and SARS (red reaction product) in pneumocytes (c, arrow). PU.1/SARS double labeling demonstrates expression of PU.1 (brown reaction product) in macrophages, most of which did not stain for SARS (red reaction product), and SARS mostly in pneumocytes negative for PU.1 (d). Weak staining for SARS was

(proliferative) phase. Taken together with the presence of extensive hyaline membrane formation, this finding points to the acute phase of DAD as the temporal window in which viral protein is most likely to be detected in the lungs of SARS-CoV-2 infected individuals. In keeping with this observation, both patients with the highest load of SARS-CoV-2-infected cells (cases 4 and 5) showed florid pneumocyte hyperplasia, with viral protein detectable within this cell population (Supplementary Fig. 1).

In patients 6 and 7, who had the longest interval between respiratory failure (as defined by the time of ICU admission), autopsy pathologic examination demonstrated organizing DAD, few to no hyaline membranes, and negative SARS IHC. Overall, the different stages of DAD observed histologically correspond to the estimated time interval from onset of respiratory failure to death; however, the exact timing of severe lung injury may be difficult to determine in certain cases given reports of silent hypoxemia in COVID-19 infected patients [27, 28]. The clearing of virus may also be affected by interventions such as remdesivir therapy [29].

SARS-CoV-2 was detected in ciliated tracheal epithelial cells and submucous glands only in the two patients (i.e., 4
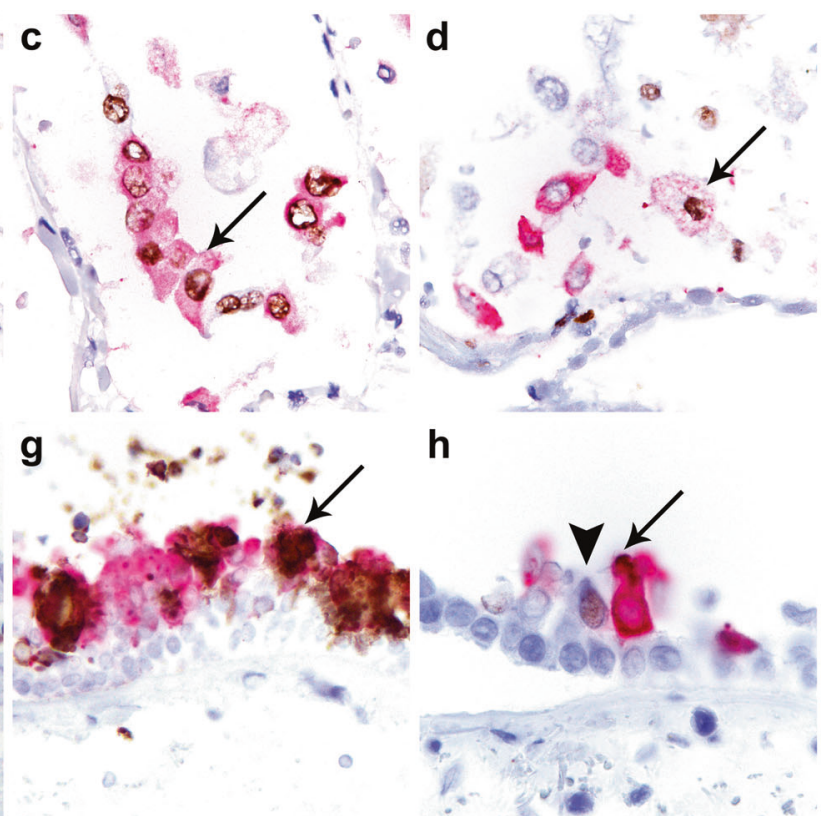

identified only in scattered macrophages (d, arrow). SARS IHC on cross sections of trachea (e) demonstrates positive staining predominantly in ciliated cells (e, arrows). P63/SARS double labeling demonstrates nuclear expression of p63 in basal cells (brown reaction product) (f, arrow) which did not stain for SARS (red reaction product). MUC5AC/SARS double labeling shows cytoplasmic expression of MUC5AC in secretory cells (brown reaction product) (g, arrow) most of which did not stain for SARS (red reaction product). FOXJ1 highlighted uninfected ciliated cells (h, arrowhead) but was absent in SARS-expressing cells (h, arrow).

and 5) with highest load of infected cells in the lung. Ciliated cells universally express the transcription factor FOXJ1 [30], however those cells showing SARS nucleocapsid positivity consistently showed absence of FOXJ1 nuclear expression. Downregulation of FOXJ1 expression in ciliated cells has been reported in airways of patients infected with Paramyxovirus [31], Respiratory Syncytial virus [32], and in mouse model of murine Cytomegalovirus infection [33], suggesting similar mechanisms of virally mediated downregulation of certain factors essential for cellular differentiation.

Overall, SARS protein was more readily detected in lung pneumocytes than airway epithelium, suggesting longer persistence of SARS-CoV-2 in pneumocytes and/or viral migration from upper to lower respiratory tract by the time of death. Within the lung, injury repair and organization appear to coincide with clearance of virally infected pneumocytes.

The cohort of patients studied here presented with typical symptoms of COVID-19 and all had comorbidities identified as risk factors for a severe clinical course. Three patients in our study received hydroxychloroquine and remdesivir [29, 34]; no distinctive histopathologic changes were observed in the treated patients. 
While our observations indicate an association between the duration from onset of respiratory failure and SARSCoV-2 abundance in the lung, many of the clinical features of this infection complicate our understanding of this relationship, including its predilection for infecting individuals with multiple comorbidities and the phenomenon of silent hypoxemia. The apparently low burden of viral protein in three of the five patients with predominantly acute-phase DAD may reflect a relatively long duration of infection prior to death, however this is necessarily speculative. Patient 1 reported 2 weeks of symptoms prior to presentation and therefore likely had begun clearing the infection from the lungs by the time of death, whereas for two patients (cases 2 and 3) with neurologic impairment, the timing of symptom onset was uncertain. Alternatively, individual variation in viral abundance may reflect specific host factors; indeed, patient 4 was profoundly immunocompromised, likely explaining the persistence of viral protein despite relatively longer duration from diagnosis to death. Finally, patient 5 had an intervening acute myocardial infarction, thus we were able to examine her virally infected lungs at an early phase of DAD.

In line with previous reports of venous thromboembolism caused by SARS-CoV-2 infection [10, 12], five patients in our cohort exhibited blood clots in pulmonary vasculature. Hypercoagulability is a common feature of DAD in general and reflects a systemic inflammatory response [35]-it is unclear whether this finding is specifically related to COVID-19 infection. However, emerging evidence suggests a higher frequency of alveolar capillary microthrombi in the context of COVID-19 than in respiratory failure due to influenza. In a recently reported autopsy series comparing lung findings in patients with COVID-19 with those from patients with influenza A (H1N1) virus and uninfected controls [12], the authors describe distinctive vascular features in lungs from patients with COVID-19, which included severe endothelial injury associated with the presence of intracellular virus and disrupted cell membranes, as well as widespread thrombosis with microangiopathy, similar to the observations made in five patients in our cohort. In comparison with patients with influenza, alveolar capillary microthrombi were significantly more prevalent in patients with COVID-19, although ACE2positive endothelial cells are observed in similar numbers in both contexts [12]. While we were not able to identify SARS-CoV-2 in endothelial cells, we cannot exclude that more sensitive techniques, such as high-resolution immunofluorescence imaging or electron microscopy, might identify additional infected cell types; more studies on larger case series are required to address this question.

We specifically restricted this study to patients with COVID-19 confirmed by RT-PCR on nasopharyngeal swab samples. While RT-PCR on respiratory tract specimens is the most commonly used diagnostic test for SARS-CoV-2 infection, its reliability is challenged by multiple clinical and technical factors [15]. Indeed, negative RT-PCR results have been reported in as many as $34.7 \%$ of CT-positive COVID-19 patients [36], highlighting the need for additional tissue-based diagnostic tests for patients with suspected COVID-19 but negative RT-PCR results. Given the absence of viral cytopathic changes in this infection, SARS IHC may be a valuable tool in this pursuit; our findings demonstrate the greatest value for SARS IHC when testing lung tissues from patients in the acute phase of DAD. In addition, our observations suggest that SARS IHC may be a helpful prospective diagnostic tool in surgical biopsy and cytology specimens, given appropriate validation. Further testing is needed to assess possible correlations between detection of SARS nucleocapsid protein by IHC and viral RNA by RT-PCR in lung tissue.

In conclusion, we demonstrate that SARS-CoV-2 predominantly localizes to airway and pulmonary epithelial cells in patients with COVID-19. The findings indicate viral migration over time. The findings suggest that SARS-CoV-2 nucleocapsid protein is detectable in the lower respiratory tract in the acute phase of DAD (within a week of onset of respiratory failure) and is absent in the organizing phase. Additional studies are required to further define organ-specific cellular tropism and SARS$\mathrm{CoV}-2$ infection dynamics over time.

Acknowledgements The authors would like to thank Mei Zheng, Immunohistochemistry Laboratory for antibody validation and staining. The authors also acknowledge Michelle Siciliano, Jacob Plaisted, and John Grzyb of the Autopsy Division for their dedication and expertise in safely performing the technical aspects of the autopsies.

\section{Compliance with ethical standards}

Conflict of interest IMS was funded by US National Institutes of Health (NIH/NCI grant K08-CA241085). SK received funding support from PhAST Diagnostics. JLH is a consultant to Epizyme, Eli Lilly, Aadi Bioscience, and TRACON Pharmaceuticals. LMS reports consulting fees/honoraria from LOXO Oncology, EMD Serono, Foghorn Therapeutics, and Astra Zeneca and research funding to the institution from Roche/Genentech. The remaining authors have no conflicts to disclose.

Publisher's note Springer Nature remains neutral with regard to jurisdictional claims in published maps and institutional affiliations.

\section{References}

1. Coronaviridae Study Group of the International Committee on Taxonomy of $\mathrm{V}$. The species severe acute respiratory syndromerelated coronavirus: classifying 2019-nCoV and naming it SARSCoV-2. Nat Microbiol. 2020;5:536-44.

2. Guan WJ, Ni ZY, Hu Y, Liang WH, Ou CQ, He JX, et al. Clinical characteristics of coronavirus disease 2019 in China. N Engl J Med. 2020;18:1708-20. 
3. Yang X, Yu Y, Xu J, Shu H, Xia J, Liu H, et al. Clinical course and outcomes of critically ill patients with SARS-CoV-2 pneumonia in Wuhan, China: a single-centered, retrospective, observational study. Lancet Respir Med. 2020;8:475-81.

4. Goyal P, Choi JJ, Pinheiro LC, Schenck EJ, Chen R, Jabri A, et al. Clinical characteristics of Covid-19 in New York City. N Engl J Med. 2020;382:2372-4.

5. Tian S, Hu W, Niu L, Liu H, Xu H, Xiao SY. Pulmonary pathology of early-phase 2019 novel coronavirus (COVID-19) pneumonia in two patients with lung cancer. J Thorac Oncol. 2020;5:700-4.

6. Zhang H, Zhou P, Wei Y, Yue H, Wang Y, Hu M, et al. Histopathologic changes and SARS-CoV-2 immunostaining in the lung of a patient with COVID-19. Ann Intern Med. 2020;172:629-32.

7. Xu Z, Shi L, Wang Y, Zhang J, Huang L, Zhang C, et al. Pathological findings of COVID-19 associated with acute respiratory distress syndrome. Lancet Respir Med. 2020;8:420-2.

8. Menter T, Haslbauer JD, Nienhold R, Savic S, Hopfer H, Deigendesch N, et al. Post-mortem examination of COVID19 patients reveals diffuse alveolar damage with severe capillary congestion and variegated findings of lungs and other organs suggesting vascular dysfunction. Histopathology. 2020; in press.

9. Barton LM, Duval EJ, Stroberg E, Ghosh S, Mukhopadhyay S. COVID-19 Autopsies, Oklahoma, USA. Am J Clin Pathol. 2020;153:725-33.

10. Wichmann D, Sperhake JP, Lutgehetmann M, Steurer S, Edler C, Heinemann A, et al. Autopsy findings and venous thromboembolism in patients with COVID-19: A Prospective Cohort Study. Ann Intern Med; 2020; in press.

11. Lax SF, Skok K, Zechner P, Kessler HH, Kaufmann N, Koelblinger $\mathrm{C}$, et al. Pulmonary arterial thrombosis in COVID-19 with fatal outcome: results from a prospective, single-center, clinicopathologic case series. Ann Intern Med. 2020; in press.

12. Ackermann M, Verleden SE, Kuehnel M, Haverich A, Welte T, Laenger $\mathrm{F}$, et al. Pulmonary vascular endothelialitis, thrombosis, and angiogenesis in Covid-19. N Engl J Med. 2020; in press.

13. Pujadas E, Ibeh N, Hernandez MM, Waluszko A, Sidorenko T, Flores V, et al. Comparison of SARS-CoV-2 Detection from Nasopharyngeal Swab Samples by the Roche Cobas 6800 SARSCoV-2 test and a laboratory-developed real-time RT-PCR test. J Med Virol. 2020; in press.

14. Zhao R, Li M, Song H, Chen J, Ren W, Feng Y, et al. Early detection of SARS-CoV-2 antibodies in COVID-19 patients as a serologic marker of infection. Clin Infect Dis. 2020; in press.

15. Sethuraman N, Jeremiah SS, Ryo A. Interpreting diagnostic tests for SARS-CoV-2. JAMA. 2020; in press.

16. Amanat F, Stadlbauer D, Strohmeier S, Nguyen THO, Chromikova V, McMahon M, et al. A serological assay to detect SARSCoV-2 seroconversion in humans. Nat Med. 2020; in press.

17. Long QX, Liu BZ, Deng HJ, Wu GC, Deng K, Chen YK, et al. Antibody responses to SARS-CoV-2 in patients with COVID-19. Nat Med. 2020; in press.

18. Harenberg J, Favaloro E. COVID-19: progression of disease and intravascular coagulation-present status and future perspectives. Clin Chem Lab Med. 2020;58:1029-36.

19. Sungnak W, Huang N, Becavin C, Berg M, Queen R, Litvinukova $\mathrm{M}$, et al. SARS-CoV-2 entry factors are highly expressed in nasal epithelial cells together with innate immune genes. Nat Med. 2020;26:681-7.
20. Munster VJ, Feldmann F, Williamson BN, van Doremalen N, Perez-Perez L, Schulz J, et al. Respiratory disease in rhesus macaques inoculated with SARS-CoV-2. Nature. 2020; in press.

21. Martines RB, Ritter JM, Matkovic E, Gary J, Bollweg BC, Bullock H, et al. Pathology and pathogenesis of SARS-CoV-2 associated with fatal coronavirus disease, United States. Emerg Infect Dis. 2020; in press.

22. Chandrashekar A, Liu J, Martinot AJ, McMahan K, Mercado NB, Peter L, et al. SARS-CoV-2 infection protects against rechallenge in rhesus macaques. Science. 2020; in press.

23. Nicholls JM, Butany J, Poon LL, Chan KH, Beh SL, Poutanen S, et al. Time course and cellular localization of SARS-CoV nucleoprotein and RNA in lungs from fatal cases of SARS. PLoS Med. 2006;3:e27.

24. Varga Z, Flammer AJ, Steiger P, Haberecker M, Andermatt R, Zinkernagel AS, et al. Endothelial cell infection and endotheliitis in COVID-19. Lancet. 2020;395:1417-8.

25. Guzik TJ, Mohiddin SA, Dimarco A, Patel V, Savvatis K, MarelliBerg FM, et al. COVID-19 and the cardiovascular system: implications for risk assessment, diagnosis, and treatment options. Cardiovasc Res. 2020; in press.

26. Merad M, Martin JC. Pathological inflammation in patients with COVID-19: a key role for monocytes and macrophages. Nat Rev Immunol. 2020;20:355-62.

27. Ottestad W, Seim M, Maehlen JO. COVID-19 with silent hypoxemia. Tidsskr Nor Laegeforen 2020;140.

28. Wilkerson RG, Adler JD, Shah NG, Brown R. Silent hypoxia: a harbinger of clinical deterioration in patients with COVID-19. Am J Emerg Med. 2020; in press.

29. Grein J, Ohmagari N, Shin D, Diaz G, Asperges E, Castagna A, et al. Compassionate use of remdesivir for patients with severe Covid-19. N Engl J Med. 2020;382:2327-36.

30. Coy S, Du Z, Sheu SH, Woo T, Rodriguez FJ, Kieran MW, et al. Distinct patterns of primary and motile cilia in Rathke's cleft cysts and craniopharyngioma subtypes. Mod Pathol. 2016;29:1446-59.

31. Look DC, Walter MJ, Williamson MR, Pang L, You Y, Sreshta $\mathrm{JN}$, et al. Effects of paramyxoviral infection on airway epithelial cell Foxj1 expression, ciliogenesis, and mucociliary function. Am J Pathol. 2001;159:2055-69.

32. Mata M, Sarrion I, Armengot M, Carda C, Martinez I, Melero JA, et al. Respiratory syncytial virus inhibits ciliagenesis in differentiated normal human bronchial epithelial cells: effectiveness of N-acetylcysteine. PLoS ONE. 2012;7:e48037.

33. Wu CA, Peluso JJ, Shanley JD, Puddington L, Thrall RS. Murine cytomegalovirus influences Foxj1 expression, ciliogenesis, and mucus plugging in mice with allergic airway disease. Am J Pathol. 2008;172:714-24.

34. Geleris J, Sun Y, Platt J, Zucker J, Baldwin M, Hripcsak G, et al. Observational Study of Hydroxychloroquine in Hospitalized Patients with Covid-19. N Engl J Med. 2020; in press.

35. Tomashefski JF Jr., Davies P, Boggis C, Greene R, Zapol WM, Reid LM. The pulmonary vascular lesions of the adult respiratory distress syndrome. Am J Pathol. 1983;112:112-26.

36. Ai T, Yang Z, Hou H, Zhan C, Chen C, Lv W, et al. Correlation of chest CT and RT-PCR testing in coronavirus disease 2019 (COVID-19) in China: A Report of 1014 Cases. Radiology. 2020; in press. 